\title{
Morfología y anatomía de ejes caulinares, licofilos y esporangios de Phlegmariurus phylicifolius: un aporte a la sistemática de las Lycopodiaceae neotropicales
}

\author{
Marcelo Daniel Arana ${ }^{1}$, Herminda Reinoso $^{2}$ \& Antonia Josefina Oggero ${ }^{1}$ \\ 1. Orientación Plantas Vasculares, Departamento de Ciencias Naturales, Facultad de Ciencias Exactas, Físico-Químicas \\ y Naturales, Universidad Nacional de Río Cuarto, Ruta 36km 601, X5804ZAB, Río Cuarto, Provincia de Córdoba, \\ República Argentina; marana@exa.unrc.edu.ar, aoggero@exa.unrc.edu.ar \\ 2. Morfología vegetal, Departamento de Ciencias Naturales, Facultad de Ciencias Exactas, Físico-Químicas y Naturales, \\ Universidad Nacional de Río Cuarto, Ruta 36km 601, X5804ZAB, Río Cuarto, Provincia de Córdoba, República \\ Argentina; hreinoso@exa.unrc.edu.ar
}

\section{Recibido 17-XII-2013. Corregido 04-IV-2014. Aceptado 05-V-2014.}

\begin{abstract}
Morphology and anatomy of caulinar axes, lycophylls and sporangia of Phlegmariurus phylicifolius: a contribution to the systematics of Neotropical Lycopodiaceae. Phlegmariurus is the only genus of Lycopodiaceae with the species grouped in 22 informal groups. Species level relationships within Phlegmariurus are poorly understood and their circumscriptions require a thorough molecular and morphological review. A detailed study of morphology and anatomy of caulinar axes, lycophylls and sporangia of Phlegmariurus phylicifolius was carried out in order to contribute to the elucidation of species circumscription in the informal group Phlegmariurus phlegmaria. Small pieces of caulinar axes bearing trophophylls, sporophylls and sporangia were fixed, dehydrated, Histowax (paraffin) embedded, sectioned in a rotatory microtome, and stained using the common Safranin O-Fast Green technique; handmade cross sections were also made and stained with the same technique. P. phylicifolius includes slender, pendulous plants up to $40 \mathrm{~cm}$ long. Shoots heterophyllous, in the basal divisions ca. 10-20(-25)mm in diameter including the trophophylls, then abruptly constricted to (1-) $1.5-2(-2.5) \mathrm{mm}$ in diameter including the imbricate, reduced sporophylls. Trophophylls are borne in alternating whorls of three, or decussate, subdecussate, or alternate, widely spaced in alternate leaved caulinar axes portions, perpendicular to the caulinar axes to falcately ascending, lanceolate to linear-lanceolate, with flat to slightly revolute margins. Each lycophyll is supplied by a single central vascular bundle, connected to a protoxylem pole in the stele. At the site of leaf-trace departure, no leaf (lycophyll) gap is present. Caulinar axes excluding leaves $0.7-1.2 \mathrm{~mm}$ thick at the base, upward tapering to ca. $0.5 \mathrm{~mm}$. Caulinar axes present unistratified epidermis and endodermis, the cortex is characterized by the presence of a trabecular structure of lisigenous origin formed in the parenchimatous tissue next to the endodermis. The vascular tissue occupies the central part of the caulinar axes, forming a plectostele of subradiate organization, with five poles of protoxylem. The epidermal cells present sinuous anticlinal walls; invaginations in the inner side of external periclinal wall of the epidermal cells could be probably adaptive morphological feature of a water deficient environment. Leaves of constricted terminal divisions are decussate, or subdecussate, continuously or discontinuously sporangiate, appressed, abaxially rounded to carinate, widely lanceolate to widely ovate or subcordate, acute to mucronate or cuspidate, shorter than the sporangia. Each sporangium originates from a group of epidermal cells, axilar to the sporophylls. The cell walls of epidermal cell of the sporangia are Huperzioideae type. The morphological studies of trophophylls contribute to confirm the differences between P. phylicifolius and P. subulatus. Rev. Biol. Trop. 62 (3): 1217-1227. Epub 2014 September 01.
\end{abstract}

Key words: caulinar axes, Huperzioideae, lycophylls, Lycopodiaceae, morphology/anatomy, Phlegmariurus, sporangia.

Lycopodiaceae es una familia compuesta por tres subfamilias: Huperzioideae, Lycopodielloideae y Lycopodioideae (Wagner \&
Beitel, 1992; Øllgaard, 2012), 16 géneros y aproximadamente 450 especies de hierbas terrestres, de distribución mundial. América 
del Sur es una de las regiones con mayor diversidad, con alrededor de 190 especies. El género Phlegmariurus (Herter) Holub presenta un alto porcentaje de endemismos, por ejemplo en los Andes, de las 95 especies citadas 69 son endémicas. Phlegmariurus es el único género de la familia dividido en 22 grupos informales y con especies poco definidas (Øllgaard, 1987; Field \& Bostock, 2013), de los cuales 11 están representados en la región Neotropical. Las principales sinapomorfías de este género son las raíces corticales, producidas en las partes distales del tallo, migrando por la corteza para emerger en las zonas de contacto con el sustrato; los esporofilos fotosintéticos persistentes luego de la dehiscencia del esporangio y esporas con lados convexos $\mathrm{o} \pm$ planos a la altura del ecuador y ángulos obtuso-agudos (Arana et al., 2012). Las características morfológicas de los licofilos en este género son de suma importancia para la caracterización de las especies, por ejemplo, Øllgaard (1987) agrupó las especies en homofilas, gradualmente heterofilas y heterofilas. Dentro de la última categoría, el grupo Phlegmariurus phlegmaria, se caracteriza por estar compuesto por plantas principalmente epifíticas y péndulas, con licofilos fuertemente dimórficos. La mayoría de las especies de este grupo son paleotropicales, estando representadas en el neotrópico por 12-16 especies, las que conforman cuatro subgrupos (Øllgaard, 1987). Análisis moleculares (Wikström, Kenrick \& Chase, 1999; Wikström \& Kenrick, 2000) sugieren que el grupo Phlegmariurus phlegmaria es polifilético, formado por dos clados sin relación entre ellos (el clado paleotropical y el neotropical) que reúnen especies con convergencia evolutiva.

En Argentina se han encontrado 22 taxones de Lycopodiaceae, reuniendo Phlegmariurus siete especies, de las cuales Phlegmariurus phylicifolius (Poiret) B. Øllg. se ha citado recientemente para el mencionado país (Arana \& Øllgaard, 2012; Arana et al., 2012). Esta especie ha sido confundida previamente con $P$. subulatus (Poiret) B. Øllg. (Rolleri, 1972; de la Sota, 1977; Rolleri, 2008) que es una especie menos tolerante a condiciones ambientales para su crecimiento, estando restringida a los bosques húmedos de los Andes, desde Costa Rica hasta Bolivia (Øllgaard, 1994; Arana \& Øllgaard, 2012). Por otra parte, P. phylicifolius está estrechamente relacionada con $P$. biformis (Hook.) B. Øllg. y P. erythrocaulon (Fée) B. Øllg. de Brasil, con las cuales forman un complejo de especies muy difíciles de separar (Øllgaard, 1992). Las entidades específicas mencionadas, más otras cinco, conforman el subgrupo Phlegmariurus myrsinites, que incluye plantas epífitas laxas con licofilos decusados o subdecusados y con tallos generalmente de color rojo.

De acuerdo con lo mencionado por $\mathrm{Ji}$, Huo, Wang y Pan (2008) y Field y Bostock (2013) en cuanto a que las relaciones a nivel de especie de Phlegmariurus están muy pobremente entendidas y su circunscripción requiere profundos estudios y revisiones, tanto moleculares como morfológicas, el objetivo de este trabajo es contribuir al conocimiento del grupo Phlegmariurus phlegmaria, con la descripción morfológica y anatómica de los ejes caulinares, trofofilos, esporofilos y esporangios de $P$. phylicifolius. De esta manera se pretende ampliar el conocimiento de las especies neotropicales del grupo y aportar a su delimitación y redefinición.

\section{MATERIAL Y MÉTODOS}

Se trabajó con material conservado en los herbarios argentinos CTES, JUA, LIL, LP, RCV y SI (Thiers, 2013) y con plantas colectadas en las Yungas de Jujuy (Argentina), entre los 1300 y $4000 \mathrm{~m}$ de altitud, las que fueron fijadas en FAA (etanol, ácido acético, formol, y agua 50:5:10:35 v/v). Los ejemplares de referencia fueron depositados en los herbarios JUA, LP y RCV. Para el estudio anatómico, muestras de ejes caulinares, trofofilos, esporofilos y esporangios se deshidrataron en una serie de mezclas de alcohol etílico-xilol, se incluyeron en Histowax (parafina) y se cortaron en sentido longitudinal y transversal a $13 \mu \mathrm{m}$ de espesor con un micrótomo rotativo. Posteriormente, las secciones fueron coloreados con la triple 
coloración de Hematoxilina-Safranina y Verde Rápido (Johansen, 1940) y se montaron utilizando el medio sintético DPX.

Se hicieron preparados semipermanentes, diafanizados con $\mathrm{NaOH}$ al $5 \%$ y preparados frescos a mano alzada de ejes caulinares y de material macerado; para ello las muestras se colocaron en una solución de $\mathrm{KOH}$ al $5 \%$ y se dejaron reposar en estufa a $60^{\circ} \mathrm{C}$ durante 24 horas. Todas estas muestras fueron coloreadas utilizando Safranina/Verde Rápido y montadas en glicerina. Los preparados se conservan en la histoteca de Morfología vegetal, Universidad Nacional de Río Cuarto y fueron observados con un microscopio Zeiss Standard 16 y fotografiados con microscopio Zeiss Axiophot, con equipo de captura y digitalización de imagen AxioVision 4.3, cámara AxioCam HRc. Para la terminología especializada se utilizó Font-Quer (1993) y Lellinger (2002).

\section{Material representativo examinado}

ARGENTINA. Jujuy. Depto. Ledesma, Parque Nacional Calilegua, Ganem, Arana \& Luna 40 (JUA, LP, RCV*). Salta. Depto. Santa Victoria, Baritú, 14-VII-1999, Ganem et al. 131 (JUA, LP, MCNS). Tucumán. Depto. Chicligasta, Quebrada Las Pavas, 25-III-1953, Schulz 8473 (LP); misma localidad, 23-III1953, Meyer 18011 (LP); Venturi 3368 (LIL, $\mathrm{SI})$.

BOLIVIA. Depto. La Paz. Prov. Franz Tamayo. Gimenez 2811, Fuentes \& Hurtado (CTES). Depto. Santa Cruz. Meereshöh, Steinbach 8450 (LIL). Depto. Cochabamba. Sacaba, 7-X-1921, Steinbach 5813 (LIL).

ECUADOR. Azuay. 16-VII-1945, Camp 4235A (CTES).

PERÚ. Depto. Puno. Carabaya, 4/5-VIII1972, Vargas 10741 (LIL).

\section{RESULTADOS}

La especie $P$. phylicifolius habita desde Costa Rica hasta el Noroeste de Argentina, e incluye plantas epífitas, péndulas, con tallos hasta de $40 \mathrm{~cm}$ de longitud, ramificados dicotómicamente, especialmente en el extremo apical, solamente radicantes en la base; las ramas poseen las partes fértiles terminales y bien diferenciadas de las estériles, bi-tri furcadas. Presenta licofilos dimorfos, los trofofilos se disponen distantes entre ellos, patentes o algo ascendentes, en general decusados o a veces reunidos en subverticilos de tres, perpendiculares al tallo o falcadamente ascendentes; angostamente lanceolados, con la base ensanchada y atenuados hacia el ápice, márgenes planos y ápice agudo, con nervadura visible, de $8-15 \times 1.5-2 \mathrm{~mm}$.

Los trofofilos presentan epidermis uniestratificada en ambas caras, las células epidérmicas son irregulares en vista superficial, alargadas y en su mayoría se disponen con el eje mayor paralelo al eje mayor de la hoja; las paredes anticlinales son sinuosas (Fig. 1A). Las hojas son hipoestomáticas, con estomas anomociticos (Fig. 1C) distribuidos en toda la superficie y ausentes en la nervadura (Fig. 1B). En corte transversal se observa que la pared periclinal externa de las células epidérmicas está engrosada, tanto en la superficie adaxial como la abaxial. Los estomas se disponen al mismo nivel que las restantes células epidérmicas y hacia el interior del mesofilo presentan una cámara subestomática amplia. El mesofilo presenta parénquima clorofílico lacunoso con células braciformes que determinan amplios espacios intercelulares (Fig. 1E). En la zona central de las hojas se observa una única nervadura que parte del sistema vascular del tallo sin laguna foliar (Fig. 1E y Fig. 1F). Rodeando al tejido vascular se encuentra una endodermis cuyas células son pequeñas y de paredes engrosadas, luego más externamente se observan varios estratos de células isodiamétricas de parénquima incoloro que se disponen de manera compacta (Fig. 1E). En material macerado se observaron sólo elementos traqueales del protoxilema, los que presentan espesamientos anillados (Fig. 1D). Las células del floema son escasas, estrechas y alargadas.

El eje caulinar posee un diámetro de 0.7$1.2 \mathrm{~mm}$, de color verde a rojizo. En corte transversal muestra una epidermis uniestratificada 

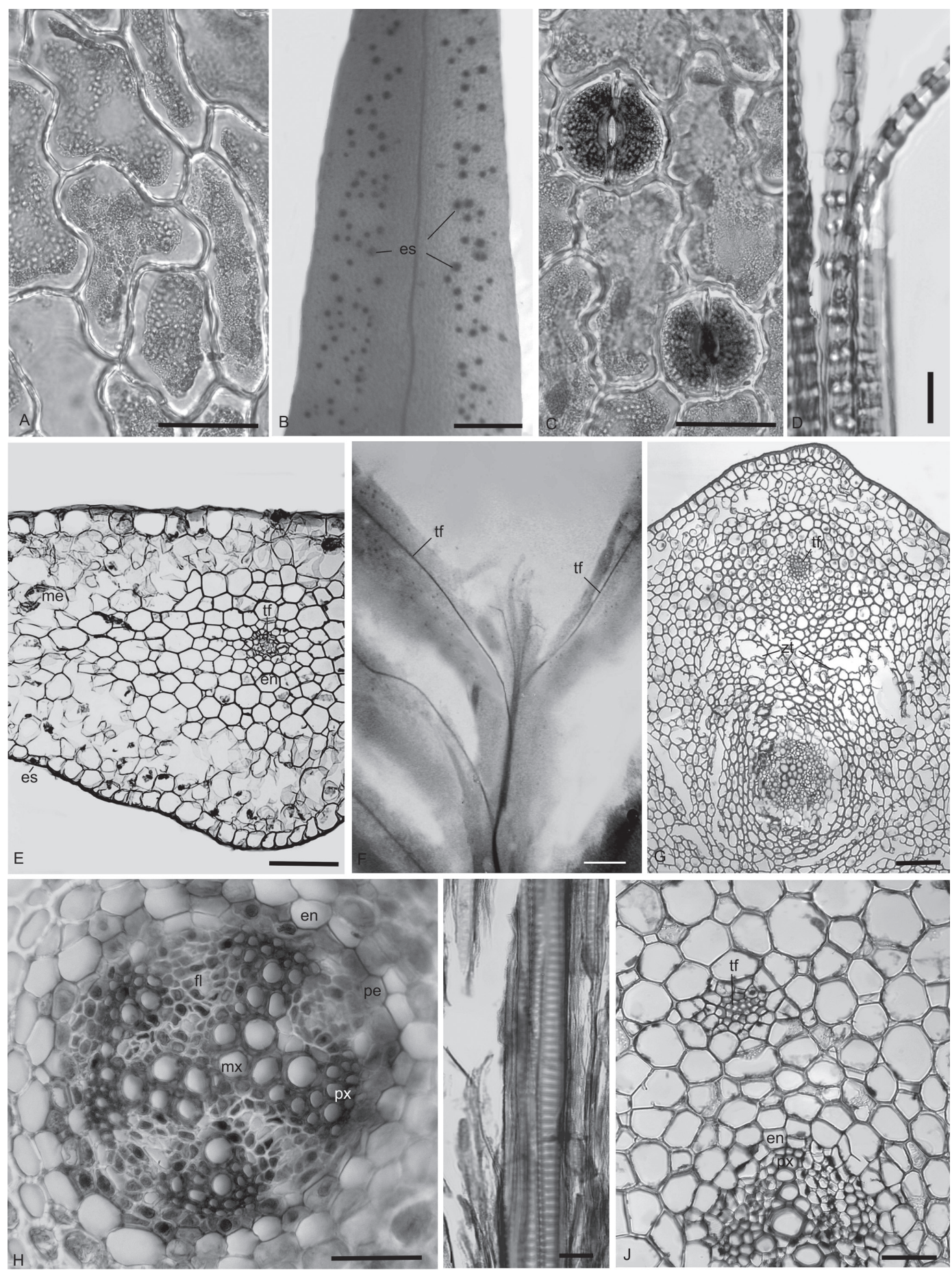

Fig. 1. Características anatómicas de los órganos vegetativos de Phlegmariurus phylicifolius. A-E licofilo. (A) vista superficial de epidermis adaxial de lámina foliar, (B) vista superficial de la epidermis abaxial de lámina foliar, (C) detalle de estomas, (D) vista longitudinal de elementos traqueales del protoxilema foliar, (E) corte transversal de lámina foliar, (F) vista longitudinal eje diafanizado mostrando la conexión vascular con los licofilos. G-J eje caulinar (G) corte transversal 
cuyas células poseen la pared tangencial externa engrosada. Hacia el interior se observa una amplia corteza de células parenquimáticas con paredes secundarias celulósicas (con la triple tinción presentan color celeste), los primeros estratos subepidérmicos corticales están dispuestos de manera homogénea y más compacta que los próximos a la endodermis que rodea al cilindro central, donde presentan una estructura trabecular (Fig. 1G). La endodermis está formada por una sola hilera de células cuyas paredes radiales y tangencial interna están levemente más engrosadas que las restantes. Entre el cilindro central y la endodermis se encuentra una/dos capas de periciclo; el tejido vascular está dispuesto en forma radial irregular (Ogura, 1972) conformando una plectostela de organización subradiada con cinco polos de protoxilema (Fig. 1H). En material macerado se observa que los elementos traqueales del protoxilema son de menor diámetro que los del metaxilema y presentan engrosamientos anillados de pared secundaria, mientras que los de metaxilema muestran punteaduras escalariformes (Fig. 1I).

Las trazas foliares emergen aparentemente del periciclo y opuestas a los polos de protoxilema aumentando su tamaño a medida que se aproximan a la superficie del eje caulinar en dirección al licofilo (Fig. 1J).

Los esporofilos son adpresos, más pequeños que los trofofilos, 1.7-2(-4)mm long, 1-1.5mm ancho, subdecusados o dispuestos en tres-cuatro hileras, ovados, cuculados, con ápice obtuso a agudo, algunas veces mucronado o cuspidado, igualando o apenas cubriendo casi completamente los esporangios, los que siempre son visibles (Fig. 2A). Los esporofilos se agrupan en áreas fértiles terminales de ramificación dicotómica. Los esporangios son axilares sobre la cara adaxial del esporofilo y no reciben vascularización, el haz foliar pasa por la base del pedicelo pero no penetra en él.

Los esporangios comienzan a formarse en el ápice caulinar junto con los primordios de los esporofilos. A medida que el tallo crece van madurando los esporangios en forma basípeta, mientras que otros nuevos se van formando del ápice. El primordio de un esporangio se observa como una pequeña protuberancia que surge por divisiones periclinales de un grupo de células epidérmicas en la región basal y adaxial de los esporofilos en formación (Fig. 2B). En corte longitudinal se observa que los esporangios desarrollan una porción apical, en forma de cabezuela que es la región fértil del mismo. La pared de esta cabezuela está constituida por tres o cuatro capas celulares (siendo la más interna el tapete) que delimitan un lóculo donde se diferencian las células madres de las esporas (CME). El esporangio presenta también un pedicelo basal que lo mantiene unido a la axila del esporofilo (Fig. 2C). Un corte trasversal por la zona fértil permite observar que cada esporangio es unilocular, pero bilobulado en la base, con los lóbulos están unidos por un tejido conectivo parenquimático. También se puede apreciar que la estructura del esporofilo es similar a la de los trofofilos, con una nervadura que ocupa la parte central. El mesofilo está constituido por parénquima clorofílico esponjoso con amplios espacios intercelulares; rodeando a la nervadura se pueden observar

\footnotetext{
del eje caulinar, aspecto general. (H) detalle de G mostrando la disposición de los tejidos vasculares. (I) vista longitudinal de elementos traqueales del metaxilema con punteaduras escalariformes. (J) sector de eje caulinar mostrando traza foliar. Abreviaturas: en, endodermis; es, estoma; fl, floema, me, mesofilo; mx, metaxilema, pe, periciclo, px, protoxilema, tf, traza foliar; zt, zona trabecular. Bar: $50 \mu \mathrm{m}$ para A, C, D, G, H e I, $400 \mu \mathrm{m}$ para B, $200 \mu \mathrm{m}$ para E y $800 \mu \mathrm{m}$ para F.

Fig. 1. Anatomical features of vegetative organs of Phlegmariurus phylicifolius. A-E lycophyll (A) surface view of adaxial epidermis of lycophyll. (B) surface view of abaxial epidermis of lycophyll. (C) detail showing stomata. (D) longitudinal view of protoxylem tracheary elements from lycophyll. (E) cross section of trophophyll. F-I rhizome (F) cross section of the stem. (G) detail of F showing the arrangement of vascular tissue $(\mathbf{H})$ longitudinal view of metaxylem cells scalariformly pitted. (I) cross section of stem showing the foliar trace. en, endodermis; es, stomata; fl, phloem, me, mesophyll; mx, metaxylem, pe, pericicle, px, protoxylem, tf, foliar trace; zt, trabecular zone. Bar: $50 \mu \mathrm{m}$ for A, C, D, G, H and I, $400 \mu \mathrm{m}$ for $\mathrm{B}$ and $200 \mu \mathrm{m}$ for $\mathrm{E}$ and $\mathrm{F}$.
} 


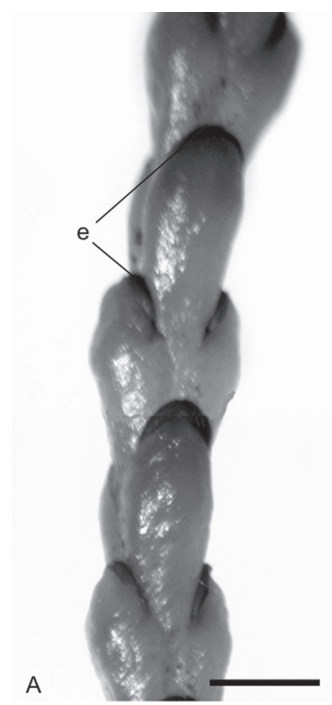

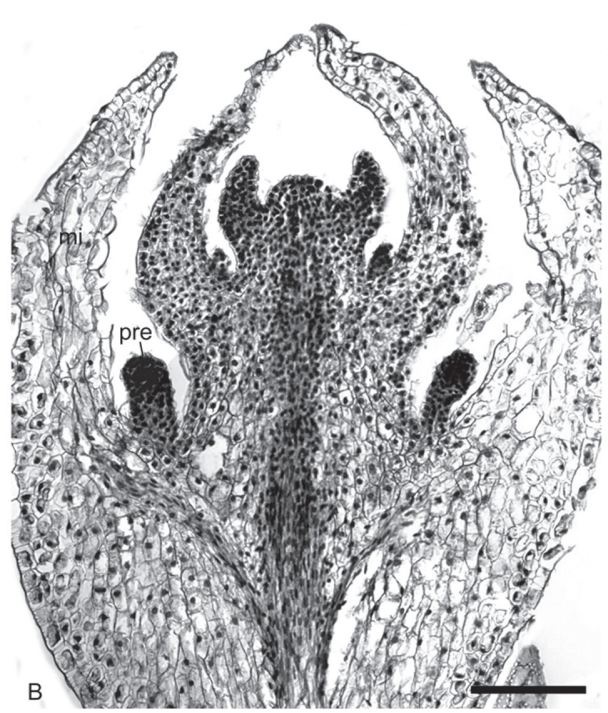
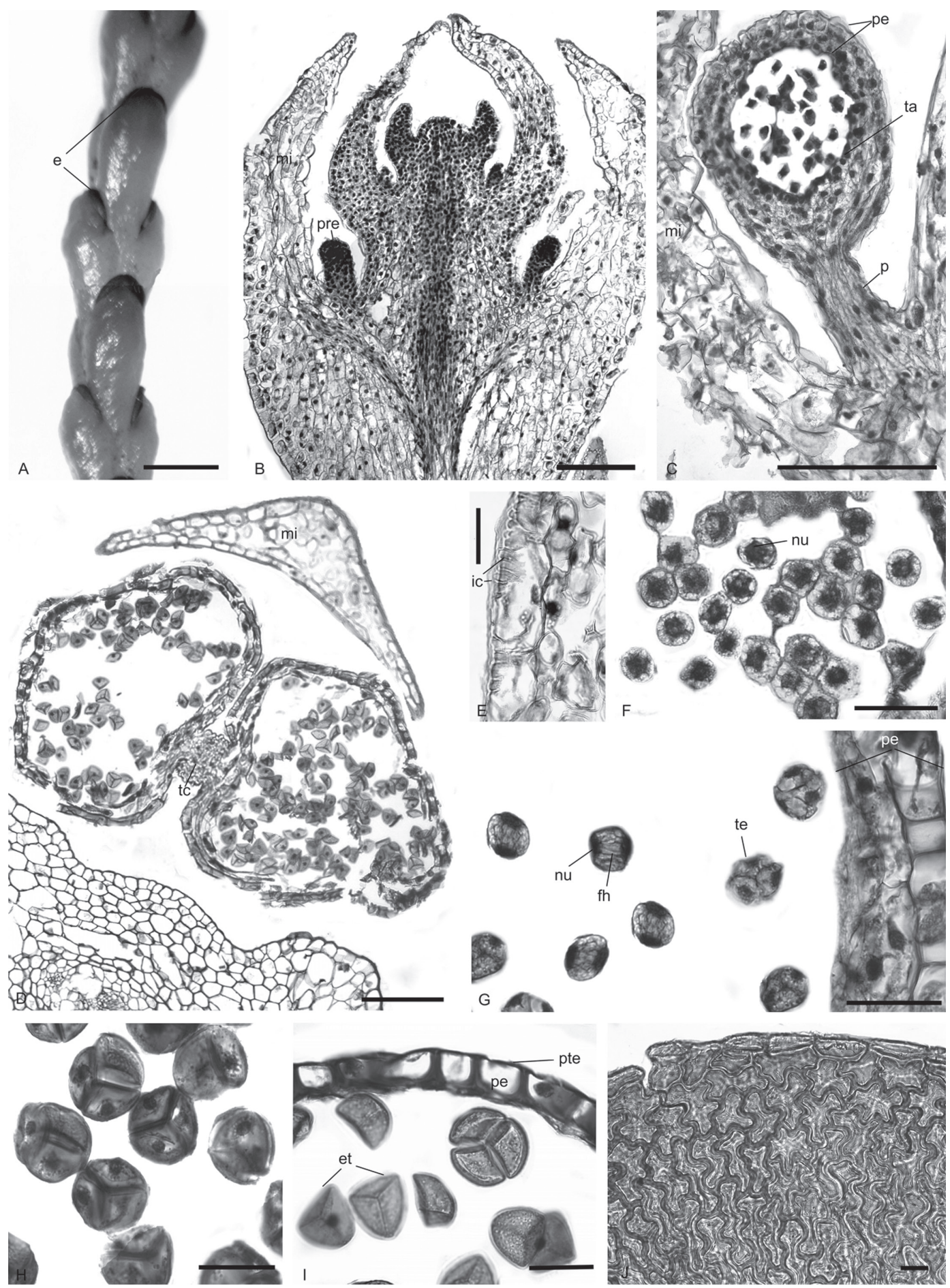

Fig. 2. Características anatómicas de las estructuras reproductivas, en distintas etapas de la esporogénesis en Phlegmariurus phylicifolius. (A) rama reproductiva mostrando la disposición de los esporofilos adpresos que protegen los esporangios. (B) corte longitudinal de ápice caulinar donde se observan los primordios de los esporangios. (C) Detalle de un esporangio 
escasas células parenquimáticas. La pared tangencial externa de las células epidérmicas es gruesa y presenta notables proyecciones que forman invaginaciones celulósicas (Fig. 2E).

Las CME se separan entre sí previo a sufrir meiosis, se hinchan y redondean y presentan un núcleo grande e irregular (Fig. 2F). Luego de experimentar la meiosis I, quedan en estado de díada, sin pared celular que separe los núcleos. En esta etapa se observan remanentes de las fibras del huso; posteriormente ocurre la cariocinesis meiotica II y se produce una citocinesis simultánea, resultando una tétrada con esporas en disposición tetraédrica (Fig. 2G). Las tétradas en su maduración incrementan su tamaño y sufren un engrosamiento progresivo del esporodermo (Fig. 2H). Si bien el tapete se diferencia durante la formación de las CME, se mantiene íntegro hasta la formación de las tétradas luego paulatinamente desaparece por degradación celular. En las tétradas maduras se pueden observar los núcleos de las esporas, finalmente las esporas triletes se liberan de la tétrada en el lóculo, donde perduran hasta la dehiscencia del esporangio (Fig. 2I).

En las etapas finales de la maduración del esporangio, en la pared se ha perdido el tapete y las capas adyacentes, quedando solo las células que constituyen el estrato celular externo, con engrosamientos lignificados (determinándose esto debido a la coloración roja intensa) en las paredes anticlinales y periclinal interna (Fig. 2I), en vista superficial, las células de los esporangios poseen paredes sinuosas y engrosadas (Fig. 2J).

\section{DISCUSIÓN}

Las licofitas actuales son los representantes de un antiguo grupo de plantas, que en el caso de las Lycopodiaceae, su origen puede rastrearse, en el paleozoico tardío (Kenrick \& Crane, 1997). En esta familia, el género Huperzia Bernh. reúne los estados de caracteres plesiomórficos (Wagner \& Beitel 1992; Wikström et al., 1999), por ejemplo H. lucidula (Michx.) Trevis., morfológicamente es prácticamente indistinguible de Drepanophycus Göpp., un linaje de licofitas del Devónico (Friedman \& Cook, 2000). Los caracteres de importancia en las Lycopodiaceae son la morfología y anatomía de la corteza, estelar, licofilos, esporas y el número de cromosomas (Øllgaard, 1992). En Huperzia, el cilindro vascular del eje caulinar constituye una actinostela en toda su longitud (Øllgaard, 1987), con el protoxilema en las terminaciones de las ramificaciones xilemáticas, mientras que el floema se localiza entre estas ramificaciones. A la madurez, las traqueidas del

en desarrollo. (D) corte transversal de un brote reproductivo mostrando un esporofilo y los lóbulos basales del esporangio. (E) detalle de la epidermis adaxial de un esporofilo mostrando las invaginaciones celulósicas en la pared. (F) esporocitos premeióticos. (G) díada al final de la meiosis I; se observan las fibras del huso meiótico y la ausencia de pared entre los núcleos. (H) tétrades con desarrollo progresivo de pared. (I) esporas triletes liberándose de la tétrade y pared del esporangio representada por un solo estrato de células mostrando sus paredes engrosadas. (J) epidermis esporangial. Abreviaturas: e, esporangio; et, espora; fh, fibras del huso; mi, esporofilo; nu, núcleo; ic, invaginación celulósica; p, pedicelo; pe, pared del esporangio; pre, primordio esporangial; pte, pared tangencial externa; tc, tejido conectivo; te, tétrade. Bar: $1 \mathrm{~mm}$ para A, $200 \mu \mathrm{m}$ para B, C, D y $50 \mu \mathrm{m}$ para E, F, G, H, I y J.

Fig. 2. Anatomical features of reproductive structures in different stages of the sporogenesis in Phlegmariurus phylicifolius. (A) reproductive shoot showing adpressed sporophylls protecting sporangia. (B) longitudinal section of apical part of reproductive shoot where sporangia primordia are evident. (C) detail of immature sporangia. (D) transversal section of reproductive shoot showing a sporophyll and the basal lobes of the sporangium. (E) Detail of adaxial epidermis of a sporophyll showing the cellulosic invaginations of the cell wall. (F) pre-meiotic sporocytes. (G) End of the meiosis I, with a dyad stage, fibers of meiotic spindle and absence of cell wall separating nuclei. (H) Tetrads in different developmental stages; view of the progressive thickening of the spore's wall. (I) Trilete spores releasing from the tetrad, sporangium wall with one cell layer, thickenings of anticlinal and inner periclinal walls becoming more evident. (J) sporangial epidermis. e: sporangium; es: stomata; et: trilete spore, fh: fibers of spindle, mi: sporophyll, nu: nucleus, ic: cellulosic invagination, p: pedicel, pe: sporangium wall, pre: primordium of the sporangium; pte: outer tangential wall, tc: connective tissue, te: tetrad. Bar: $1 \mathrm{~mm}$ for A, $200 \mu \mathrm{m}$ for B, C and D, $50 \mu \mathrm{m}$ for E, F, G, H, I and $\mathrm{J}$. 
protoxilema exhiben engrosamientos parietales anulares, que ocasionalmente se interconectan con engrosamientos verticales formando un retículo laxo (Friedman \& Cook, 2000). En $P$. phylicifolius se observa una plectostela pentaradiada, con el protoxilema en los polos de los radios xilemáticos, coincidiendo en líneas generales con lo observado por Rolleri (1972). Este tipo de plectostela radial parece ser un rasgo muy constante en las Lycopodiaceae (Ogura, 1972, Øllgaard, 1987). Una particularidad de $P$. phylicifolius es el desarrollo temprano de trabéculas de origen lisígeno, en donde se observan restos de paredes celulares, que se forma en los estratos parenquimáticos de la corteza próximos a la endodermis. Esta estructura trabecular ha sido observada previamente en especies terrestres como $P$. saururus (Lam.) B. Øllg., aunque con origen esquizógeno, generalmente asociado a especies con ejes caulinares crasos o suculentos.

Una característica particular en los géneros Huperzia y Phlegmariurus (Huperzioideae), es la presencia de raíces corticales internas, que crecen paralelas a la estela de los ejes caulinares y emergen en las zonas de contacto con el sustrato (Arana \& Øllgaard, 2012). De acuerdo a Stokey (1907) estas raíces están relacionadas con el hábito erecto de las especies, colaborando con la sustentación de las plantas. Estas estructuras radicales no fueron observadas en $P$. phylicifolius, esta ausencia probablemente está asociada más al hábito péndulo de estas plantas que al epifitismo, ya que estas porciones corticales de las raíces han sido registradas en Lycopodiaceae epífitas de crecimiento erecto como P. mandiocanus (Raddi) B. Øllg. y P. pithyoides (Schlecht. \& Cham.) B.Øllg., (Ogura, 1972; Kessler, 2009).

Otras características asociadas al crecimiento erecto de la planta es la turgencia de las células de la corteza y tejido lignificado en el eje caulinar, aunque ésta última característica no siempre estaría asociada al hábito de la planta (Pita, De Menezes \& Prado, 2006). Se han encontrado especies epífitas de Phlegmariurus, en Brasil, que poseen tejidos colenquimatosos y lignificados en las capas externas de la corteza. Esta característica no se ha observado en $P$. phylicifolius, donde la corteza está formada íntegramente por tejido parenquimático, sin lignificación ni presencia de colénquima; lo que confirmaría la estrecha relación con el hábito péndulo epífito característico de esta especie.

$P$. phylicifolius crece en los bosques yungueños donde hay períodos de escasez hídrica y de nutrientes de forma marcada. Esta especie crece sobre los troncos, por lo cual su nutrición depende del acúmulo de detritos, que está asociado a las características de la corteza del forofito (Pita et al., 2006). Su hábito de crecimiento varía fuertemente de acuerdo a las condiciones ambientales (Øllgaard, 1994). En el material estudiado, los trofofilos poseen su ancho máximo en la base, lo que corresponde con las características del tipo de Lycopodium nubigenum Herzog, y que se observa en las plantas provenientes del área sur de la distribución (Perú, Bolivia y Argentina); en cambio, en plantas provenientes de Ecuador los trofofilos poseen su ancho máximo en su región media, tentativamente se mantiene a L. nubigenum como sinónimo de $P$. phylicifolius, requiriéndose estudios más profundos. Por otra parte, este trabajo contribuye a confirmar las diferencias entre $P$. phylicifolius y $P$. subulatus, ya que en ésta última los trofofilos son lineal subulados, de $6-15 \mathrm{~mm}$ de longitud y $0.5-1 \mathrm{~mm}$ de ancho; mientras que en $P$. phylicifolius son lanceolados a lineal-lanceolados, de 8-13(-15)mm de longitud y $1.5-2 \mathrm{~mm}$ de ancho.

Las trazas foliares se originan junto a los polos de protoxilema $\mathrm{y}$, aparentemente se forman a partir del periciclo, con escasos elementos vasculares diferenciados; a medida que se aproxima a la base foliar, la cantidad de elementos traqueales aumenta, estando ya diferenciados en la zona media del licofilo.

De acuerdo a Esau (1985) la variación en las invaginaciones celulósicas de las células epidérmicas puede deberse a factores ambientales, prevaleciendo las invaginaciones en ambientes más húmedos (Rolleri, 1972; Pita et al., 2006). En la especie estudiada, las invaginaciones están formadas en la pared primaria. 
Estas invaginaciones se registraron en $P$. friburgensis (Nessel) B. Øllg. (Pita et al., 2006), que crece sobre sustratos rocosos, pobres en nutrientes y con poca capacidad de retención de agua. Allí se registra un período de sequía de cuatro a cinco meses, característica compartida por los ambientes donde crece $P$. phylicifolius. Por ello consideramos que las invaginaciones podrían estar relacionadas con los períodos de aridez estacional, brindándole a la planta un refuerzo para disminuir la posibilidad de plasmólisis en épocas desfavorales. Los trofofilos son hipostomáticos, con los estomas dispuestos por toda la epidermis, aunque están ausentes en proximidades de la nervadura, sin formar bandas estomáticas como lo observado en otra especie epífita simpátrica, $P$. mandiocanus. Esto podría deberse a que la última posee los licofilos lineales, con muy poca superficie efectiva. Estos estomas poseen un desarrollo acrópeto y corresponden al tipo perígeno, al igual que lo observado por Rolleri (1972) para otras especies.

Con respecto a la heterofilia, la especie estudiada presenta esporofilos diferenciados de los trofofilos, siendo los primeros reducidos y no fotosintéticos; este estado de carácter es considerado derivado dentro del género Phlegmariurus y ocurre por reducción de esporofilos a partir de ancestros homofilos (Øllgaard, 1987), fenómeno que ha ocurrido varias veces, en forma independiente, en el curso evolutivo del linaje (Wikström et al., 1999; Wikström \& Kenrick, 2000).

En Lycopodiaceae se han descripto tres patrones morfológicos de los esporangios, con estrecha correlacion taxonómica (Øllgaard, 1975), que fueron utilizados para caracterizar las subfamilias por Arana y Øllgaard (2012) y confirmados en esta especie, que presenta un patrón de las células de la epidermis esporangial con paredes sinuosas y moderada a fuertemente lignificadas. Los esporangios comienzan a formarse, a partir de un grupo de células epidérmicas localizadas en la axila de los primordios de licofilos en el ápice caulinar. A medida que el eje caulinar crece van madurando los esporangios de posición más basal, mientras que otros nuevos se van formando del ápice, por lo que el desarrollo de los esporangios es basípeto, fenómeno observado en otras Lycopodiaceae (Rolleri, 1972; Sérsic, 1983; Øllgard 1987; Uehara \& Kurita, 1991; Rincón Barón, Gélvez Landazábal, Forero Ballesteros, Arrieta Prieto \& Hleap, 2009) y que corresponde a una sinapomorfía de la familia. Durante la ontogenia esporangial en $P$. phylicifolius, se observa que inicialmente la pared que delimita el lóculo presenta tres o cuatro capas celulares; mientras que Rolleri (1972) observó, en diferentes especies de Lycopodium, que la pared del esporangio estaba formada inicialmente por seis a siete capas celulares que se reduce a cuatro durante el desarrollo, y Rincón Barón et al. (2009) encontraron que en P. brevifolius la pared del esporangio poseía cuatro a cinco capas de células. En $P$. phylicifolius la más externa forma la pared definitiva del esporangio, las demás capas intermedias degeneran, una condición similar a la descrita por Uehara y Kurita (1991) para L. clavatum. Las células del estrato externo de la pared del esporangio adquieren engrosamientos celulósicos que luego se lignifican en las paredes anticlinales y periclinales, lo que ha sido mencionado como una característica general de la familia (Øllgaard, 1975, 1987, 1990) que se confirma en P. phylicifolius.

\section{RESUMEN}

Phlegmariurus es el único género de Lycopodiaceae con las especies reunidas en 22 grupos informales. Las relaciones a nivel de especie dentro de Phlegmariurus están pobremente estudiadas y la circunscripción de las mismas requiere profundos exámenes moleculares y morfológicos. Se ha llevado a cabo un estudio detallado de la morfología y la anatomía de ejes caulinares, licofilos y esporangios de $P$. phylicifolius, con el fin de contribuir al esclarecimiento en la delimitación de las especies en el grupo Phlegmariurus phlegmaria. Segmentos de ejes caulinares con trofofilos, esporofilos y esporangios fueron fijados, deshidratados, incluidos en Histowax (parafina), cortados con un micrótomo rotatorio y coloreados usando la técnica tradicional Safranina O-Verde Rápido; además se hicieron cortes a mano alzada y se colorearon con la misma técnica. $P$. phylicifolius incluye plantas colgantes y péndulas de hasta $40 \mathrm{~cm}$ de longitud. Los ejes son heterofilos, de aproximadamente $10-20(-25) \mathrm{mm}$ de diámetro 
en las divisiones basales incluyendo los trofofilos, luego abruptamente reducidos a (1-) $1.5-2(-2.5) \mathrm{mm}$ de diámetro incluyendo los esporofilos reducidos e imbricados. Los trofofilos están dispuestos en anillos alternantes de a tres, o decusados, subdecusados o alternos, dispuestos en forma espaciada en los ejes caulinares, perpendiculares al tallo hasta falcadamente ascendentes, lanceolados a lineallanceolados, con márgenes lisos o levemente revolutos. Cada licofilo está provisto de un haz vascular simple y central, conectado a un polo de protoxilema de la estela y sin laguna foliar. Los tallos poseen un ancho de $0.7-1.2 \mathrm{~mm}$ en la base, excluyendo los licofilos, estrechándose hasta cerca de $0.5 \mathrm{~mm}$ hacia el ápice. Los ejes caulinares presentan una epidermis uniestratificada y endodermis, la corteza se caracteriza por la presencia de una estructura trabecular de origen lisígeno formada en el tejido parenquimático próximo a la endodermis. El tejido vascular ocupa la parte central del eje caulinar, formando una plectostela de organización subradiada, con cinco polos de protoxilema. Las células epidérmicas presentan paredes anticlinales sinuosas; las invaginaciones en la cara interna de la pared periclinal externa podrían ser probablemente un característica morfológica adaptativa a un ambiente con períodos de sequía. Las hojas de las porciones apicales son decusadas o subdecusadas, con esporangio de disposición continua o discontinua, adpresas, abaxialmente redondeadas a carinadas, ampliamente lanceoladas a ovadas o subcordadas, ápice agudo a mucronado o cuspidado, más corto que el esporangio. Cada esporangio se origina de un grupo de células epidérmicas, en la axila de los esporofilos con el eje caulinar. Las paredes celulares de las células epidérmicas del esporangio son de tipo Huperzioideae. El estudio de la morfología de los trofofilos contribuye a confirmar las diferencias entre P. phylicifolius y P. subulatus.

Palabras clave: eje caulinar, esporangios, Huperzioideae, licofilos, Lycopodiaceae, morfología/anatomía, Phlegmariurus.

\section{REFERENCIAS}

Arana, M. D. \& Øllgaard, B. (2012). Revisión de las Lycopodiaceae (Embryopsida, Lycopodiidae) de Argentina y Uruguay. Darwiniana,50(2), 266-295.

Arana, M. D., Morrone, J. J., Ganem, M. A., Luna, M. L., Ramos-Giacosa, J. P., \& Giudice, G. (2012). Diversidad y análisis panbiogeográfico de las licofitas (Embryopsida: Lycopodiidae) del Parque Nacional Calilegua, Jujuy, Argentina. Iheringia (Série Botanica), 67(2), 177-188.

de la Sota, E. R. (1977). Pteridophyta, en A. L. Cabrera (Ed.), Flora de la Provincia de Jujuy (pp. 1-275). Argentina: Colección Científica del Instituto Nacional de Tecnología Agropecuaria.

Esau, K. (1985). Anatomía de las plantas con semilla. Buenos Aires: Hemisferio Sur.
Field, A. R. \& Bostock, P. D. (2013). New and existing combinations in Palaeotropical Phlegmariurus (Lycopodiaceae) and lectotypification of the type species Phlegmariurus phlegmaria (L.) T.Sen \& U.Sen. PhytoKeys, 20, 33-51.

Font-Quer, P. (1993). Diccionario de Botánica. Barcelona: Ed. Labor.

Friedman, W. E. \& Cook, M. E. (2000). The origin and early evolution of tracheids in vascular plants: integration of palaeobotanical and neobotanical data. Philosophical.Transactions of.Royal Society of London B, 355, 857-868.

Ji, S., Huo, K., Wang, J., \& Pan, S. (2008). A molecular phylogenetic study of Huperziaceae based on chloroplast $\mathrm{rbcL}$ and psbA-trnH sequences. Journal of Systematics and Evolution (Acta Phytotaxonomica Sinica), 46(2), 213-219.

Johansen, D. A. (1940). Plant Microtechnique. New York: Mc-Graw-Hill Book.

Kenrick, P. \& Crane, P. R. (1997). The origin and early diversification of land plants: A cladistic study. Washington D.C.: Smithsonian Institution Press.

Kessler, E. (2009). Esporófito de Huperzia mandiocana (Raddi) Trevisan (Lycopodiaceae): morfoanatomia e ontogênese. (Master's thesis). Universidade Federal de Santa Catarina, Brasil. Retrived from https://repositorio.ufsc.br/bitstream/ handle/123456789/92913/279704.pdf?sequence=1

Lellinger, D. B. (2002). A modern multilingual glossary for taxonomic pteridology. Pteridologia, 3, 1-263.

Ogura, Y. (1972). Comparative anatomy of vegetative organs of the pteridophytes. In W Zimmermann, S. Carlquist, R. Ozenda, H. D. Wulff (Eds.), Handbuch der Pflanzenanatomie (pp. 1-502). Berlin, Stuttgart: Bornträger.

Øllgaard, B. (1975). Studies in Lycopodiaceae I. Observations on the Structure of the Sporangium wall. American Fern Journal, 65, 19-27.

Øllgaard, B. (1987). A revised classification of the Lycopodiaceae s. lat. Opera Botanica, 92, 153-178.

Øllgaard, B. (1990). Lycopodiaceae. In K. Kubitzki (Ed.), The Families and Genera of Vascular Plants I (pp. 31-39). Berlin \& Heidelberg: Springer-Verlag.

Øllgaard, B. (1992). Neotropical Lycopodiaceae, an overview. Annals of the Missouri Botanical Garden, 79, 687-717.

Øllgaard, B. (1994). Lycopodiaceae, en R. M. Tryon, \& R. G. Stolze (Eds.), Pteridophyta of Peru, Parte VI. Fieldiana Botanica, 34, 16-66.

Øllgaard, B. (2012). New combinations in Neotropical Lycopodiaceae. Phytotaxa, 57, 10-22.

Pita, P. B., De Menezes, N. L., \& Prado, J. (2006). Morfologia externa e interna das folhas vegetativas, 
esporofilos e esporangios de espécies de Huperzia Bernh. (Lycopodiaceae - Pteridophyta) do Brasil. Revista Brasileira Botanica, 29(1), 115-131.

Rincón Barón, E. J., Gélvez Landazábal, L. V., Forero Ballesteros, H. G., Arrieta Prieto, D., \& Hleap, J. S. (2009). Ontogenia del esporangio y esporogénesis del licopodio Huperzia brevifolia (Lycopodiaceae) de las altas montañas de Colombia. Revista de Biología Tropical, 57(4), 1141-1152.

Rolleri, C. H. (1972). Morfología comparada de las especies de Lycopodium L. (Lycopodiaceae-Pteridophyta) para el noroeste argentino. Revista del Museo de La Plata, Sección Botánica, 12, 223-383.

Rolleri, C. H. (2008). Lycopodiaceae, in F. O. Zuloaga, O. Morrone, \& M. J. Belgrano (Eds.), Catálogo de las Plantas Vasculares del Cono Sur (Argentina, Sur de Brasil, Chile, Paraguay y Uruguay). St. Louis, Mo., U.S.A.: Missouri Botanical Garden Press.

Sérsic, A. N. (1983). Ontogenia del esporangio y esporogénesis en Lycopodium saururus (Lycopodiaceae). Boletín de la Sociedad Argentina de Botánica, 22(14), 205-220
Stokey, A. G. (1907). The roots of Lycopodium pithyoides. Botanical Gazette, 44, 57-61.

Thiers, B. (2013). [continuously updated, accessed 2013] Index Herbariorum: a global directory of public herbaria and associated staff. New York Botanical Garden's Virtual Herbarium, http://sweetgum.nybg. org/ih

Uehara, K. \& Kurita, S. (1991). Ultrastructural study on spore wall morphogenesis in Lycopodium clavatum (Lycopodiaceae). American Journal of Botany, 78, 24-36.

Wagner, W. H. Jr. \& Beitel, J. M. (1992). Generic classification of modern North American Lycopodiaceae. Annals of the Missouri Botanical Garden, 79, 676-686.

Wikström, N. \& Kenrick, P. (2000). Phylogeny of epiphytic Huperzia (Lycopodiaceae): paleotropical and neotropical clades corroborated by plastid $r b c L$ sequences. Nordic Journal of Botany, 20, 165-171.

Wikström, N., Kenrick, P., \& Chase, M. (1999). Ephiphytism and terrestrialization in tropical Huperzia (Lycopodiaceae). Plant Systematics and Evolution, 218, 221-243. 
